\title{
An Improved Projection Method for Determination of Fatigue Parameters of Metal Structures Based on Spherical Direction Cosine Group Construction
}

\author{
B. Yang, ${ }^{1}$ B. Q. Ma, Y. Y. Wu, B. Chen, S. N. Xiao, G. W. Yang, and T. Zhu \\ State Key Laboratory of Traction Power, Southwest Jiaotong University, Chengdu, China \\ 1.yb@swjtu.cn
}

The key components of railway rolling stock are almost constantly in the multiaxial stress state in service. To evaluate the fatigue strength of the structure, stress parameters should be obtained beforehand. The traditional projection method assumes that the maximum principal stress is the maximum tensile stress, and then the projection process is performed without taking the tension/ compression states of principal stresses into account. To address these inadequacies, a method for fatigue parameter determination was proposed based on principal stress projection toward a spherical direction cosine group. This method can determine the direction of maximum tensile stress and preserve the tension/compression attribute of stress during calculating the maximum stress and the minimum stress. Therefore, the physical meaning of present improved method is more apparent than the traditional projection method, and its algorithm is simple and effective. The fatigue strength assessments of a welded bogie frame were performed utilizing the traditional projection method and present method respectively. Results show that some maximum stresses calculated by the improved method could be 19.4\% higher than those obtained by the traditional one at some nodes, while the minimum stresses and the stress ratio deduced from these two methods could even be of opposite sign. The present method, which takes the tension/compression properties of principal stresses into consideration, is more reasonable, as compared with the traditional projection method, and thus the fatigue strength assessment results are more credible.

Keywords: fatigue strength assessment, projection method, direction cosine group, tension/ compression properties, welded bogie frame.

Introduction. During the service process of railway rolling stock, the key structures bears complicated alternate loadings. To restrict the possible fatigue failure and to ensure the safety of railway operation, fatigue strength assessments for key components are important and essential. Commonly, these components are always in multiaxial stress state in service, so multiaxial fatigue strength theory is necessary to obtain relevant fatigue parameters, such as the maximum stress, $\sigma_{\max }$, and the minimum stress, $\sigma_{\min }$. Then fatigue assessment can be performed by utilizing fatigue limit diagrams or $S-N$ curves. Among existing multiaxial fatigue assessment methods for key components of railway rolling stock, such as wheel and bogie frame, the direct method [1], the equivalent fatigue stress method [2], the tensor method [3], the equivalent mean stress method [4], the amendatory Crossland method [5], and the projection method [6] are all applied to some degree. These methods are to make the simplification from multiaxial stress state to uniaxial stress or to equivalent stress possible, among which the projection method is most accepted and adopted in railway industry. This method is introduced in Appendix G of ERRI B12/RP17, which is provided by the European Rail Research Institute [6]. By vector coordinate transforming and stress projecting to reference direction, the projection method can perform the fatigue strength assessment of complicated structures with the Goodman diagrams.

The traditional projection method is based on the following hypothesis: when fatigue crack occurs at a point of structure, it develops perpendicular to the largest normal tensile stress. This hypothesis has been repeatedly confirmed by experience, so projection method has explicit physical meaning. However, there are also some inadequacies for this method. 
Firstly, in the practical operation, $\sigma_{\max }$ is defined directly as the largest principal tensile stress in all loading cases and its direction is regarded as the preferred direction for following projection process. Thus, the actual maximum stress and its direction may be neglected, so that $\sigma_{\max }$ is smaller than its true value. Secondly, the principal stresses come in pairs with two opposite directions, but this characteristic has not received enough attention in projection method. As a result, the projection process may confuse the sign of stress, so that the tension/compression state of projected stress is changed and the fatigue strength analysis results tend to be dangerous or over-conservative.

By constructing a spherical direction cosine group, present research can determine the direction of maximum tensile stress and preserve the tension/compression attribute of principal stress during projection process. The maximum stress and the minimum stress obtained by present method are more reasonable while the physical essence of the present improved method is more apparent than the traditional projection method.

\section{Traditional Projection Method and Its Limitation.}

1.1. Principal Assertions and Realization. The traditional projection method implies that when fatigue crack initiates at a point of structure it growths in a direction perpendicular to the largest normal tensile stress. The method thus consists in finding this "privileged" or preferred direction and in calculating the dynamic variations of the normal component of the stress applied to the material element in the preferred direction. The following procedure is adopted [5]:

(i) the principal stresses and corresponding principal directions in different loading cases are determined for some point of structure;

(ii) the largest principal tensile stress in all loading cases is defined as $\sigma_{\max }$ while its direction is chosen to be the projection direction, which is perpendicular to the preferred direction;

(iii) the normal components of the principal stresses which are exerted on the material element of preferred direction are calculated in other loading cases;

(iv) the smallest of the stress values obtained above is defined as $\sigma_{\min }$;

(v) so far the fatigue parameters of the point considered, such as the mean stress $\sigma_{m}$ and the stress amplitude $\sigma_{a}$, can be evaluated by following two equations:

$$
\begin{gathered}
\sigma_{m}=\frac{\sigma_{\max }+\sigma_{\min }}{2}, \\
\sigma_{a}=\frac{\sigma_{\max }-\sigma_{\min }}{2} .
\end{gathered}
$$

Standard of International Union of Railways, UIC 515-4 [7], and standard of British Standards Institution, BS EN 13749 [8] provide the algorithm, which is consistent with the solving idea of ERRI B12/RP17, for calculating fatigue stress parameters of bogie frame and for wheel respectively. In standard of UIC 510-5 [9], the projection process is introduced in detail. It can be briefly stated as follows: assume that $t$ loading cases are applied to the structure while $\sigma_{\max }$ occurs in case $k$ and its direction cosine is $n_{k}$. In another loading case $i$, three principal stresses and their direction cosine are described as $\sigma_{1 i}, \sigma_{2 i}, \sigma_{3 i}$ and $n_{1 i}, n_{2 i}, n_{3 i}$. Then the sum of projection values in direction of $\sigma_{\max }$ can be represented as

$$
\sigma_{e q, i}=\sigma_{1 i} n_{1 i} \cdot n_{k}+\sigma_{2 i} n_{2 i} \cdot n_{k}+\sigma_{3 i} n_{3 i} \cdot n_{k} .
$$

Then the minimum value of $\sigma_{e q, i}$ calculated from all the loading cases, except for the case $k$, is $\sigma_{\min }$. 
1.2. Limitation of the Traditional Projection Method. The inadequacies of traditional projection method lie in following two aspects:

1.2.1. Determination of the Largest Tensile Stress and Its Direction. The viewpoint in ERRI B12/RP17 that crack develops in a direction perpendicular to the largest tensile stress is widely accepted by a series of fatigue strength assessment standards for railway vehicle key components [7-11] and related academic articles [1-3, 12, 13]. However, taking the stress state of structure in typical loading case into account, the maximum first principal stress is normally defined as $\sigma_{\max }$ directly to facilitate the simplification of calculation. The direction of this stress is then set to be the projection direction for multiaxial stress conversion, while the dynamic variation of the equivalent stress in this direction is regarded as the effective parameter for fatigue strength assessment.

However, the real stress distribution of structure is quite complicated. The principal stress matrixes for the same structure point in different loading cases are different. If some principal stress is assumed to be the maximum principal stress in advance, the true largest tensile stress and its direction may be ignored. As shown in Fig. 1a, assume that for a point of structure in some loading case, three principal stresses are all equivalent tensile stresses $\left(\sigma_{1}=\sigma_{2}=\sigma_{3}\right)$ and the value is larger than any principal stress in other loading case. Then $\sigma_{1}$ is selected to be $\sigma_{\max }$ according to traditional projection method, and its direction $(1,0,0)$ is the projection direction. But, in fact, the maximum tensile stress should be the resultant stress of three principal stresses, $\sigma_{\max }=\sqrt{\sigma_{1}^{2}+\sigma_{2}^{2}+\sigma_{3}^{2}}=\sqrt{3} \sigma_{1}$, and the direction cosine is $(\sqrt{3} / 3, \sqrt{3} / 3, \sqrt{3} / 3)$. It can be seen that the tensile stress is 1.73 times the value of $\sigma_{1}$. By using the traditional projection method in this example, the real largest tensile stress is ignored, so that following assessment results may tend to be unsafe.

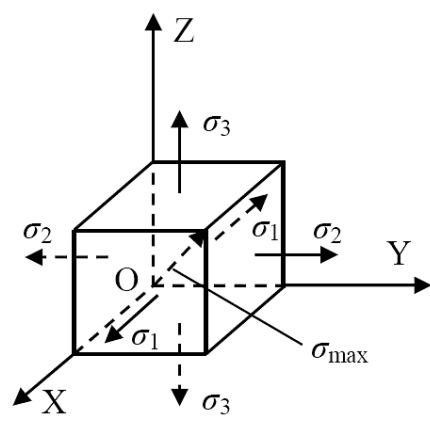

a

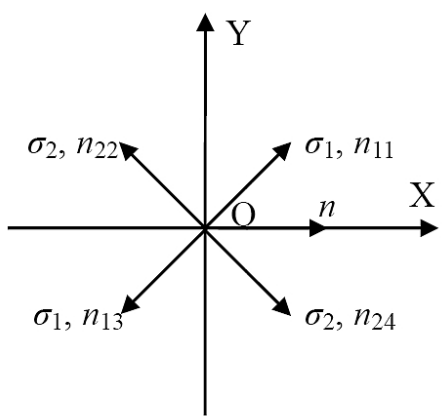

b

Fig. 1. Limitation of traditional projection method: (a) largest tensile stress; (b) tension/compression property attribute of stress.

1.2.2. Preservation of the Tension/Compression Attribute of the Principal Stress. As shown in Fig. 1b, $\sigma_{1}$ and $\sigma_{2}$ are tensile stresses of point $O$ in $X O Y$ plane. The direction cosines of $\sigma_{1}$ in first quadrant and third quadrant are $n_{11}(\sqrt{2} / 2, \sqrt{2} / 2,0)$ and $n_{13}(-\sqrt{2} / 2$, $-\sqrt{2} / 2,0)$, respectively. The direction cosines of $\sigma_{2}$ in second quadrant and fourth quadrant are $n_{22}(-\sqrt{2} / 2, \sqrt{2} / 2,0)$ and $n_{24}(\sqrt{2} / 2,-\sqrt{2} / 2,0)$, respectively. The principal stresses come in pairs with two opposite directions. But when $\sigma_{1}$ and $\sigma_{2}$ are projected to direction $n(1,0,0)$ without judging their tension/compression attribute, four different values of projection components can be obtained, i.e., $(\sqrt{2} / 2)\left(\sigma_{1}+\sigma_{2}\right),(-\sqrt{2} / 2)\left(\sigma_{1}+\sigma_{2}\right)$, $(\sqrt{2} / 2)\left(\sigma_{1}-\sigma_{2}\right)$, and $(-\sqrt{2} / 2)\left(\sigma_{1}-\sigma_{2}\right)$. Though it is intuitively plausible that $\sigma_{1}$ in third quadrant and $\sigma_{2}$ in second quadrant point to the opposite direction of $n$ and are compressive stresses, actually they have tensile effect on point $O$ as $n$ is the exterior 
normal direction. Hence, $\sigma_{1}$ in first quadrant and $\sigma_{2}$ in fourth quadrant should be chosen to perform the projection procedure. In short, the angle between principal stress to be projected and projection direction should be an acute angle so as to keep its tension/ compression attribute. Traditional projection method cannot preserve this attribute in multiaxial stress conversion, and $\sigma_{\min }$ obtained by this method may be either larger or smaller which can lead to fatigue strength assessment results incorrect.

2. Improved Method Based on Spherical Direction Cosine Group Construction. To improve above two inadequacies of traditional projection method, an improved fatigue parameter determination method was proposed in present study. It includes two key steps:

(1) Construction of spherical direction cosine group.

(2) Superposition of stress projection components and determination of maximum stress and minimum stress.

2.1. Construction of Spherical Direction Cosine Group. To some point of structure, projection direction assumed in advance may not be the true direction of largest tensile stress. So a direction cosine group is considered to be established so that the accurate projection direction is covered in this group. It is easy to perceive that the direction cosine group will form a spherical surface around the point of structure with a radius of unit length. Once any possible direction starting from this point is included in the direction cosine group, the largest tensile stress must exist in one of the directions. Construction method is introduced in detail as follows:

(i) as shown in Fig. 2a, assume that $O$ is a point of structure to be analyzed. Then create the Cartesian coordinates with the origin at point $O$;

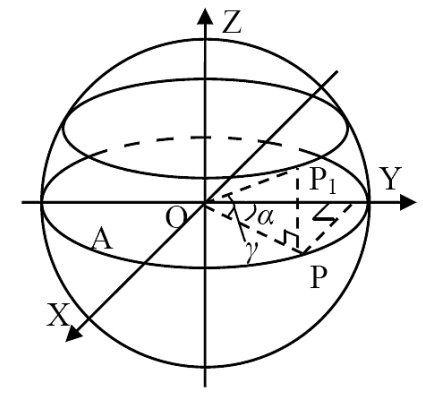

a

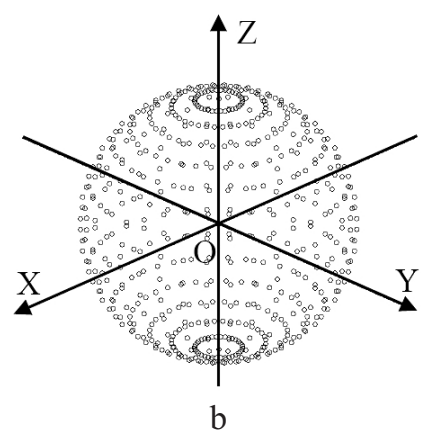

Fig. 2. Construction of spherical direction cosine group: (a) construction method; (b) a direction cosine group.

(ii) create a circle $A$ with radius of 1 in $X O Y$ plane;

(iii) take $(0,1,0)$ for the starting point, determine a series of points on the circumference of $A$ along clockwise direction or counterclockwise direction. The choice of angle interval is based on practical requirement. If $P$ is one of the points, then set the angle between $O P$ and $X$-axis to be $\alpha$. Thus the direction cosine of $O P$ is $(\sin \alpha, \cos \alpha, 0)$. Record all the direction cosines of the points on circumference in turn;

(iv) the direction cosine group is a spherical surface around original point $O$. So if cut the spherical surface with ZOP plane and set an elevation of $\gamma$ relative to XOY plane, the direction cosine $O P_{1}\left(P_{1}\right.$ is the intersection on spherical surface) can be expressed as $(\sin \alpha \times \cos \gamma, \cos \alpha \times \cos \gamma, \sin \gamma)$. Similarly, all the direction $\operatorname{cosines}$ with the same elevation of $\gamma$ and corresponding to points on the circumference of $A$ can be determined;

(v) adjust the elevation of $\gamma$ in the range of $-90^{\circ} \sim 90^{\circ}$ according to practical requirement and repeat step (4), the direction cosine group starting from point $O$ can be constructed. The size of the group can be easily controlled by adjusting the degree interval in step (3) and (4). The smaller the interval is, the greater the number of directions is. 
Therefore, the largest tensile stress obtained in following process will be closer to the actual. But meanwhile, increase of the group size will consume more time in calculating. It is necessary to make an appropriate balance between calculation efficiency and accuracy.

Figure $2 \mathrm{~b}$ illustrates a direction cosine group. Lines from origin $O$ to points on spherical surface form all the directions. For this direction cosine group, intervals of $\alpha$ and $\gamma$ are both $10^{\circ}$. Totally 612 directions are included.

2.2. Determination of the Maximum and Minimum Stresses. After establishing the direction cosine group for some point $O$ of structure, it can be applied to solve $\sigma_{\max }$ and $\sigma_{\min }$. Suppose point $O$ has went through $t$ loading cases and the number of directions for the group is $p$, project three principal stresses of $O$ in case $i$ to any direction $n_{j}$ of the group and calculate the sum of every projected stress components. To reserve the tension/compression attribute of principal stress conveniently, a new algorithm is proposed in the form of Eq. (4) to keep $n_{j}$ as the exterior normal direction,

$$
\sigma_{e q, i j}=\sigma_{1 i}\left|n_{1 i} \cdot n_{j}\right|+\sigma_{2 i}\left|n_{2 i} \cdot n_{j}\right|+\sigma_{3 i}\left|n_{3 i} \cdot n_{j}\right| .
$$

By introducing absolute value sign in Eq. (4), the problem caused by the fact that the principal stresses come in pairs with two opposite directions can be solved. During the process of projection and superposition, sign of principal stress is always retained so that its tension/compression can be reserved. This algorithm meets the requirement of angle between principal stress and the projection direction to be acute for multiaxial stress conversion. By calculating $\sigma_{e q, i j}$ of point $O$ in all $t$ loading cases and to all $p$ directions, totally $t \times p$ equivalent stresses can be derived. Then, choose the largest $\sigma_{e q, i j}$ to be $\sigma_{\max }$ and the smallest $\sigma_{e q, i j}$ in that direction to be $\sigma_{\min }$.

3. Example of Fatigue Analysis for Bogie Frame. To indicate the reasonability and the validity of present improved method, fatigue strength assessments and results comparisons were performed for bogie frame by present method and by traditional projection method respectively

3.1. Structure of Bogie Frame and Finite Element Model. Frame is the skeleton structure of the bogie. It is the installation base for different parts, and can bear and transfer various kinds of loads. The frame is a welded structure, which is composed of side beams, transoms and end beams. Q345E, which is high-strength low alloy structural steel produced in China, is used as the bogie frame material. The mechanical properties at room temperature of the material are as follows: $210,000 \mathrm{MPa}$ for the Young modulus, 0.3 for Poisson's ratio, $630 \mathrm{MPa}$ for tensile strength, and $345 \mathrm{MPa}$ for yield strength. The chemical composition can be found in [14]. Solid element is applied for the discretization of the frame. There are totally 3076,293 elements and 5589,954 nodes in the finite element model (as shown in Fig. 3).

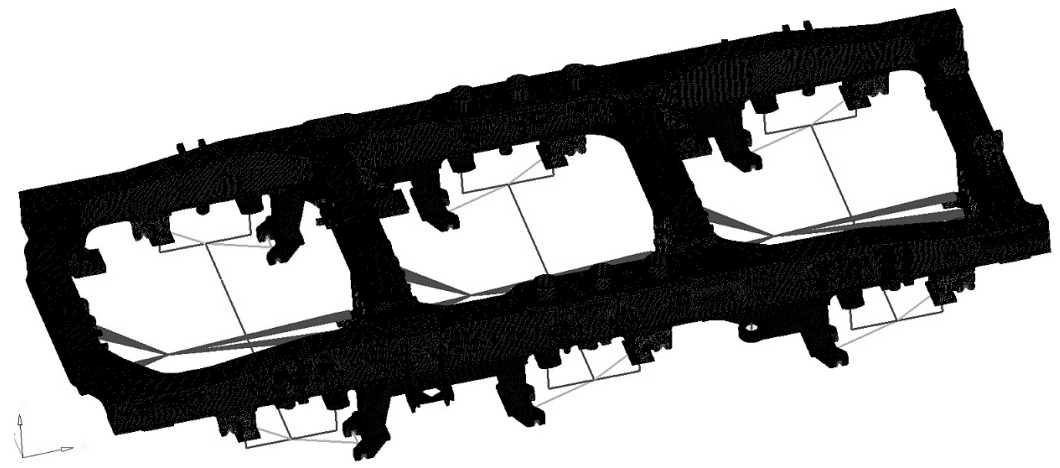

Fig. 3. Finite element model of a welded bogie frame. 
3.2. Forces, Constraints, and Loading Cases. According to UIC 615-4 [11], 13 normal service loading cases were determined as listed in Table 1, which include vertical force $F_{Z}$ applied to each side oeam, lateral force $F_{Y}$, weight of driving system, force caused by track twist, and so on. Coefficients $\alpha$ and $\beta$ are set to be 0.1 and 0.2 , respectively.

$\mathrm{T}$ a b 1 e 1

Loading Cases for Simulation of Normal Service Loads

\begin{tabular}{|c|c|c|c|c||}
\hline \multirow{2}{*}{ Case } & \multicolumn{2}{|c|}{ Vertical force applied to each side beam } & \multirow{2}{*}{ Lateral force } & Track twist \\
\cline { 2 - 3 } & $F_{Z 1}$ & $F_{Z 2}$ & & 0 \\
\hline 1 & $F_{Z}$ & $F_{Z}$ & 0 & 0 \\
\hline 2 & $(1+\alpha-\beta) F_{Z}$ & $(1-\alpha-\beta) F_{Z}$ & 0 & 0 \\
\hline 3 & $(1+\alpha-\beta) F_{Z}$ & $(1-\alpha-\beta) F_{Z}$ & $F_{Y}$ & 0 \\
\hline 4 & $(1+\alpha+\beta) F_{Z}$ & $(1-\alpha+\beta) F_{Z}$ & 0 & 0 \\
\hline 5 & $(1+\alpha+\beta) F_{Z}$ & $(1-\alpha+\beta) F_{Z}$ & $F_{Y}$ & 0 \\
\hline 6 & $(1-\alpha-\beta) F_{Z}$ & $(1+\alpha-\beta) F_{Z}$ & 0 & 0 \\
\hline 7 & $(1-\alpha-\beta) F_{Z}$ & $(1+\alpha-\beta) F_{Z}$ & $-F_{Y}$ & 0 \\
\hline 8 & $(1-\alpha+\beta) F_{Z}$ & $(1+\alpha+\beta) F_{Z}$ & 0 & 0 \\
\hline 9 & $(1-\alpha+\beta) F_{Z}$ & $(1+\alpha+\beta) F_{Z}$ & $-F_{Y}$ & $5 \% 0$ \\
\hline 10 & $(1+\alpha-\beta) F_{Z}$ & $(1-\alpha-\beta) F_{Z}$ & $F_{Y}$ & $5 \% 0$ \\
\hline 11 & $(1+\alpha+\beta) F_{Z}$ & $(1-\alpha+\beta) F_{Z}$ & $F_{Y}$ & $5 \% 0$ \\
\hline 12 & $(1-\alpha-\beta) F_{Z}$ & $(1+\alpha-\beta) F_{Z}$ & $-F_{Y}$ & $5 \% 0$ \\
\hline 13 & $(1-\alpha+\beta) F_{Z}$ & $(1+\alpha+\beta) F_{Z}$ & $-F_{Y}$ & \\
\hline
\end{tabular}

3.3. Fatigue Strength Assessment. By utilizing the APDL program, principal stresses and corresponding direction cosines of 500 nodes on bogie frame in all the 13 loading cases were extracted. Furthermore, equivalent fatigue stress parameters were calculated according to traditional projection method and present improved method respectively. Values of $\sigma_{\max }$ and $\sigma_{\min }$ of some typical nodes are listed in Table 2 while the fatigue strength assessment effect by depicting these parameters in Goodman diagram provided in ERRI B12/RP17 is shown in Fig. 4a. It is obvious that no matter which method is applied, the fatigue strength of the bogie frame can meet the requirement of standard. Though the difference between data distribution obtained by two methods is not very significant from integral view as shown in Fig. $4 \mathrm{a}$, it is necessary to note that the values of $\sigma_{\max }$ calculated by traditional projection method for 41 nodes are smaller than those obtained by present improved method. As for $\sigma_{\min }$, traditional projection method leads to more than $1 \mathrm{MPa}$ stress difference for 139 nodes to present method, among which $\sigma_{\text {min }}$ of 97 nodes are larger while $\sigma_{\min }$ of the other 42 nodes are smaller. To facilitate the understanding, a local view is illustrated in Fig. 4b to indicate the differences between fatigue parameters obtained by traditional projection method and by present method.

As listed in Table 2, for the typical nodes, there are always some differences in fatigue parameters except for node 128397. It is because $\sigma_{\max }$ is defined directly as the largest first principal stress in traditional projection method so as to the real largest tensile stress is neglected $\left(\sigma_{\max }\right.$ of node 254570 obtained by traditional method is $19.4 \%$ smaller than that obtained by present method). Furthermore, traditional projection method cannot determine the tension/compression property of principal stress, so the value or even the sign of stress component after projection could be changed. 
An Improved Projection Method for Determination of Fatigue Parameters ...

$\mathrm{T}$ a b 1 e 2

Equivalent Fatigue Stress Parameters of Typical Node

\begin{tabular}{|c|c|c|c|c||}
\hline \multirow{2}{*}{$\begin{array}{c}\text { No. } \\
\text { of node }\end{array}$} & \multicolumn{2}{|c|}{ Traditional projection method } & \multicolumn{2}{c||}{ Present method } \\
\cline { 2 - 5 } & $\sigma_{\max }$ & $\sigma_{\min }$ & $\sigma_{\max }$ & $\sigma_{\min }$ \\
\hline 124505 & 32.89 & -30.85 & 32.89 & 19.00 \\
\hline 128397 & 134.68 & 65.37 & 134.68 & 65.37 \\
\hline 128657 & 53.84 & 10.56 & 53.84 & -14.12 \\
\hline 254254 & 43.40 & -23.28 & 46.39 & 26.65 \\
\hline 254570 & 42.67 & 19.85 & 50.95 & 28.51 \\
\hline
\end{tabular}

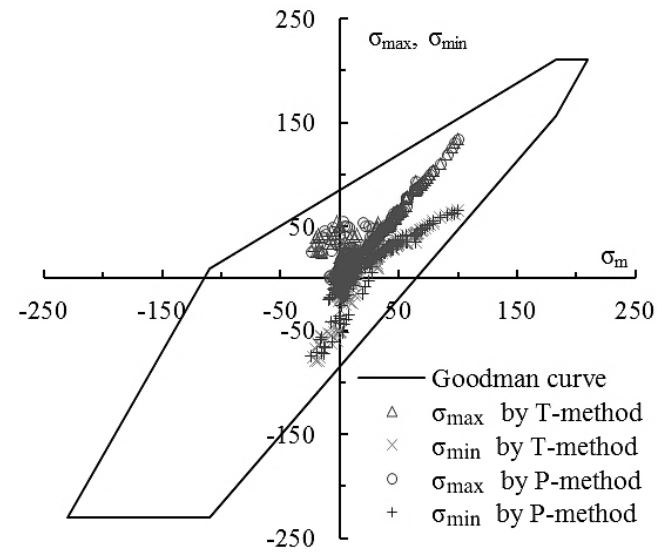

a

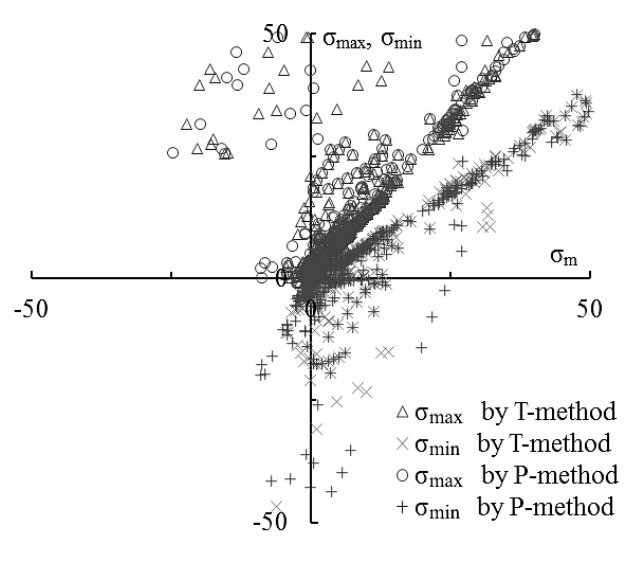

b

Fig. 4. Fatigue strength assessment effect by traditional projection method (T-method) and by the proposed improved method (P-method): (a) the integral view; (b) the local view.

Taking node 254254 as an example, Table 3 gives the principal stresses and corresponding direction cosines of this node in loading case A and loading case B. The largest stress and smallest stress occurs in case A and case B, respectively. According to traditional projection method, $\sigma_{1, A}$ in case A is the maximum stress $\sigma_{\max }$ with a value of $43.40 \mathrm{MPa}$ and its direction cosine $n_{1, A}(0.462,0.560,-0.688)$ is the projection direction. Put $\sigma_{1, B}, \sigma_{2, B}, \sigma_{3, B}$, and $n_{1, A}$ into Eq. (3), the sum of stress components is $-23.28 \mathrm{MPa}$. However, this projection process changes the tensile stress $\sigma_{1, B}$ from $24.34 \mathrm{MPa}$ to a stress component of $-24.24 \mathrm{MPa}$ for reason that the angle between $n_{1, A}$ and $n_{1, B}$ is obtuse. This compressive effect of stress component is inconsistent to practical situation.

The proposed improved method constructs a direction cosine group of node by spherical scanning. Direction $n(0.750,0.433,-0.5)$ and/or $(-0.750,-0.433,0.5)$ is searched for the direction of largest tensile stress. Then by utilizing equation (4), ?max is determined to be $46.39 \mathrm{MPa}$, which is almost $3 \mathrm{MPa}$ larger than the value obtained by traditional projection method. Also $\sigma_{\text {min }}$ can be derived to be $26.65 \mathrm{MPa}$, which is quite opposite to the previous $-23.28 \mathrm{MPa}$. As for projected stress component of $\sigma_{1, B}$, present method preserve the tensile attribute of principal stress. It is clear that the algorithm of Eq. (4) helps using the sign of principal stress to preserve its tension/compression characteristic. In addition, the values of stress ratio $R$, which is defined as $\sigma_{\min } / \sigma_{\max }$, obtained by traditional method and by improved method are -0.54 and 0.57 , respectively. 
B. Yang, B. Q. Ma, Y. Y. Wu, et al.

$\mathrm{T}$ a b 1 e 3

Principal Stresses and Corresponding Direction Cosines of Node 254254

in Different Loading Cases

\begin{tabular}{|c|c|c|c|c|c|c|c|c||}
\hline \multirow{2}{*}{$\begin{array}{c}\text { Principal } \\
\text { stress }\end{array}$} & \multicolumn{4}{|c|}{ Loading case A } & \multicolumn{4}{c||}{ Loading case B } \\
\cline { 2 - 9 } & $\sigma$ & \multicolumn{3}{|c|}{$n$} & $\sigma$ & \multicolumn{3}{c||}{$n$} \\
\hline$\sigma_{1}$ & 43.40 & 0.462 & 0.560 & -0.688 & 24.34 & -0.401 & -0.531 & 0.747 \\
\hline$\sigma_{2}$ & 16.66 & 0.799 & 0.074 & 0.597 & 10.72 & 0.856 & 0.074 & 0.512 \\
\hline$\sigma_{3}$ & 2.66 & -0.386 & 0.825 & 0.413 & 1.87 & -0.327 & 0.844 & 0.425 \\
\hline
\end{tabular}

Such significant difference is critical for determination of fatigue endurance and then for assessment of fatigue strength.

Therefore, the proposed improved projection method can yield the true largest tensile stress effectively (e.g., node 254254 and node 254570), and can avoid changing the tension/compression property of principal stress thereby preventing $\sigma_{\min }$ from being unreasonably augmented (e.g., node 128657) or diminished (e.g., node 124505 and node 254570). Moreover, the obtained value of stress ratio $R$ is more credible.

Conclusions. A fatigue parameter determination method was proposed based on principal stress projection toward a spherical direction cosine group. This method can improve the two major inadequacies of traditional projection method. Comparison of fatigue strength assessment results of a welded bogie frame show that maximum stress calculated by the improved method happen to be $19.4 \%$ higher than that obtained by the traditional one at some node, while the minimum stresses and the stress ratio deduced from these two methods can even be opposite in sign. The proposed improved projection method can determine the direction of true largest tensile stress effectively and keep the tension/ compression attribute during the projection process. It allows one to avoid ignoring the real $\sigma_{\max }$ and prevent changing the tension/compression property of principal stress thereby keeping $\sigma_{\min }$ from being unreasonably over- or underestimated. Moreover, present method can ensure more reliable estimation of the stress ratio $R$. The algorithm of present method is rather simple while its physical essence is more explicit. It can be applied to fatigue strength assessment of key components of railway rolling stock, such as wheel and bogie frame, reasonably and conveniently.

Acknowledgments. Present work is supported by the National Natural Science Foundation of China (51205326 and 51275432), the Opening Project of State Key Laboratory of Traction Power (Grant No. 2015TPL_T13), and the Fundamental Research Funds for the Central Universities (SWJTU11CX075).

1. B. R. Li and Y. X. Zhao, "Strength assessment for bogie frame based on UIC standard," Machinery, 39, 9-12 (2012).

2. H. Y. Liu, P. P. Zhang, and C. Y. Mi, "Research of design method of rolling stock wheel strengths," J. China Railway Soc., 29, 102-108 (2007).

3. S. N. Xiao, C. Yang, and G. W. Yang, "A novel fatigue analysis method of welded frames," J. Southwest Jiaotong Univ., 48, 199-204 (2013).

4. G. Sines, "Behaviour of metals under complex static and alternating stresses," in: G. Sines and J. L. Waisman (Eds.), Metal Fatigue, McGraw-Hill, New York (1959), pp. 145-169.

5. P. P. Zhang, J. C. Liu, and B. Zhang, et al., "Assessment method for fatigue strength of EMU wheel based on principal stress method and amendatory crossland method," China Railway Sci., 35, 52-57 (2014). 
6. ERRI B12/RP 17-1997. Programme of Tests to Be Carried Out on Wagons with Steel Underframe and Body Structure (Suitable for being Fitted with the Automatic Buffing and Draw Coupler) and on Their Cast Steel Frame Bogies, 8th edition, European Rail Research Institute, Utrecht (1997).

7. UIC 515-4: 1993. Passenger Rolling Stock - Trailer Bogies - Running Gear-Bogie Frame Structure Strength Tests, International Union of Railways, Paris (1993).

8. BS EN 13749: 2011. Railway Applications. Wheelsets and Bogies. Methods of Specifying Structural Requirements of Bogie Frames, British Standards Institution, London (2011).

9. UIC 510-5: 2007. Technical Approval of Monobloc Wheels, International Union of Railways, Paris (2007).

10. TB/T 2368: 2005. Motive Power Unit - Bogies and Running Gear - Bogie Frame Structure Strength Tests, China Ministry of Railway, Beijing (2005).

11. UIC 615-4: 2003. Motive Power Units - Bogies and Running Gear - Bogie Frame Structure Strength Tests, International Union of Railways, Paris (2003).

12. J. Lu, C. Y. Mi, Y. J. Liu, and G. Li, "Research on wheel parametric design based on ANSYS and fatigue post-processing system," Electr. Drive Locomotiv., Issue 6, 32-35 (2013).

13. B. Yang and Y. X. Zhao, "Experimental research on dominant effective short fatigue crack behavior for railway LZ50 axle steel," Int. J. Fatigue, 35, 71-78 (2012).

14. GB/T 1591: 2008. High Strength Low Alloy Structural Steels, China National Standardization Management Committee, Beijing (2008). 